\title{
A Hybrid Genetic Algorithm for Component Sequencing and
}

\section{Feeder Arrangement}

\author{
William HO and Ping JI* \\ Department of Industrial and Systems Engineering \\ The Hong Kong Polytechnic University \\ Hung Hom, Kowloon, Hong Kong \\ FAX: (852)23625267, E-MAIL: MFPJI@POLYU.EDU.HK
}

\begin{abstract}
:
This paper presents a hybrid genetic algorithm to optimize the sequence of component placements on a printed circuit board and the arrangement of component types to feeders simultaneously for a pick-and-place machine with multiple stationary feeders, a fixed board table and a movable placement head. The objective of the problem is to minimize the total traveling distance, or the traveling time, of the placement head. The genetic algorithm developed in the paper hybridizes different search heuristics including the nearest neighbor heuristic, the 2-opt heuristic, and an iterated swap procedure, which is a new improving heuristic. Compared with the results obtained by other researchers, the performance of the hybrid genetic algorithm is superior to others in terms of the distance traveled by the placement head.
\end{abstract}

Keywords: Genetic algorithms, heuristics, printed circuit board manufacturing, surface mount technology, component placement sequencing

*Author to whom all correspondence should be addressed. 


\section{Introduction}

Enormous growth of demands from consumers, commercial and industrial computers and other electronic devices such as mobile phones and industrial robots has made Printed Circuit Board (PCB) assembly become a fast growing market. Moreover, the requirements from consumers, such as small product size, more functions and high reliability, have forced the Surface Mount Technology (SMT) to replace the pin-through-hole (PTH) technology since the SMT has enabled the mounting of a quite large number of electronic components on a single PCB. In order to improve the performance of placement machinery in terms of speed and accuracy, the circuit board manufacturing industry relies more and more upon highly automated production processes including the numerically controlled machines for the placement of SMT components on the circuit board. The expense of the automated assembly equipment motivates PCB manufacturers to plan for the optimal assembly process that either minimizes the total production cost or maximizes the profit.

Since the placement operation is the most time consuming among the assembly operations and has the greatest potential on production cost reduction, many researchers have investigated the problems related to the placement operation. The placement operation consists of the following three basic steps or sub-problems (Ball and Magazine, 1988):

(i) allocation of components to machines,

(ii) allocation of which types of components to feeders at each machine,

(iii) pick and placement sequencing.

In this paper, the second and the third sub-problems, in the SMT environment, are studied. For the PTH technology, the sequence of the insertion operation in the auto-insertion machine can be simply formulated as the traveling salesman problem (Chan and Mercier, 1989), and it is unnecessary to consider the feeder arrangement problem. However, in the SMT environment, the sequence of components to be put on the PCB is dependent on the feeder location to hold 
which types of components. If the arrangement of components to feeders is not made carefully, even the pick and placement sequencing is solved for optimality, it can result in an extremely poor performance (Altinkemer et al., 2000). So, certainly, the problem of component placement sequencing as well as the feeder arrangement should be solved simultaneously.

However, it was observed that many researchers solved the problems for the pick-and-place machine, being studied in this paper, separately. Ball and Magazine (1988) modeled the PCB component sequencing problem for a moving head, a stationary board table, stationary feeders machine, that is, a pick-and-place (PAP) machine, with a given feeder arrangement as the rural postman problem. They used heuristics to combine the three previously-mentioned problems into two sub-problems and solved them with the balance and connect heuristic, which assured the solution to be optimal if the assembly head movement was rectilinear. Ji et al. (1992) studied the component sequencing and feeder arrangement problems for the PAP machine. The problems were formulated as two linear assignment problems, each solved with the reduced matrix method. Foulds and Hamacher (1993) determined the sequence of component placements and feeder arrangement for the PAP machine. The feeder arrangement problem, which was formulated as a number of one-facility location models, was solved first. Then, the component sequencing problem, which was formulated as the traveling salesman problem, was solved afterwards. Francis et al. (1994) studied the component sequencing and feeder arrangement problems for the PAP machine. The problems were formulated as the traveling salesman problem in order to minimize the total assembly time. A heuristic approach was developed to solve the problems. Kumar and Li (1995) determined the sequence of component placements as well as the feeder arrangement for the PAP machine. Although the authors formulated the problems as an integer quadratic programming model, they did not solve it to optimality because they found that the model was computationally intractable. Therefore, they solved the problems separately. First, the 
component sequencing problem was referred as the traveling salesman problem (TSP). The nearest neighbor, the nearest insertion, the furthest insertion, and a random generation were used to generate an initial sequence. Then, 2-optimality, 3-optimality, and or-optimality heuristics were used to improve the initial sequence. Second, the feeder arrangement problem was referred as the minimum weight matching problem (MWMP). They used commercial software to obtain an optimal solution for the MWMP. Broad et al. (1996) focused on the problems of component sequencing and feeder arrangement for the PAP machine. The authors formulated the problems as an integer linear programming model. They adopted the solution procedure, which was developed by other researchers, to solve the feeder arrangement problem first and then the component sequencing problem. Magyar et al. (1999) applied several search heuristics to solve the component sequencing and feeder arrangement problems separately for the PAP machine. The authors first determined the feeder arrangement, and then the order of component placements.

Unlike the above approaches, the problem here is to determine the sequence of component placements and the assignment of component types to feeders at the same time for the PAP machine. Naturally, the problem itself is extremely difficult to solve. On the other hand, in the recent years, Genetic Algorithms (GA) have been applied successfully to a wide variety of optimization problems such as the QAP, the minimum spanning tree problem, and the TSP (Davis, 1991; Chambers, 1995; Mitchell, 1996; Gen and Cheng, 1997). The success is critical due to GA’s simplicity, ease of operation, and great flexibility. However, it was found that none of the researchers have studied the combined problem for the PAP machine by using a hybrid GA (HGA). So, this paper proposes a HGA to solve the problem. The algorithm incorporates with three heuristics, namely, the nearest neighbor heuristic (NNH), the 2-opt local search heuristic (Reinelt, 1994), and a newly developed heuristic called the iterated swap procedure (ISP). 
This paper is organized as follows. Section 2 provides a brief overview of two types of PCB assembly machines. Section 3 describes the hybrid GA for the combined problem of component sequencing and feeder arrangement. Section 4 discusses the result from the hybrid GA, and compares it with the results obtained by Leu et al. (1993) and Ong and Khoo (1999). Section 5 concludes the paper.

\section{PCB assembly machines}

In the SMT environment, there are mainly two types of placement machines. Each type of machines possesses its own characteristics as well as operations. The first type of machines is called the chip shooter machine. It has a moving feeder carrier, a moving $\mathrm{X}-\mathrm{Y}$ table carrying a PCB, and a rotary turret having multiple assembly units. Each assembly unit has several nozzles with different sizes. A large nozzle is used to pick up and place large components. The major advantage of the chip shooter machine is its high speed. However, it is only preferable to operate with small components such as chip resistors.

The second type of machines, being studied in this paper, is called the pick-and-place (PAP) machine. In this type of machines, the components are stored in multiple stationary feeders. The placement head travels to pick up a component from a feeder at a time, and then place it on the stationary board. Although the speed of the PAP machine is slower than that of the chip shooter machine, the PAP machine is able to achieve higher accuracy, and suitable to operate with large components such as Integrated Circuit (IC). The operation sequence of the PAP machine is that the placement head starts from its original location (starting point or home position), moves to a feeder that carries components, picks a component from the feeder, then moves to the desired placement location on the stationary board, and places it there. After that, the head moves back to the previous feeder if the next component is the same type with the previous one or moves to another feeder if it is different to pick up another component and 
repeats the operation procedure. After completing all component placements on a board, the head returns to its original location, and waits for the next board to be assembled. Since the combined problem of component sequencing and feeder arrangement is for this type of machines only, the problem can be regarded as the PAP problem (Leu et al., 1993).

\section{Hybrid genetic algorithm}

Genetic Algorithms (GA) are stochastic optimization techniques (Davis, 1991; Chambers, 1995; Mitchell, 1996; Gen and Cheng, 1997). A GA starts with an initial set of random solutions, called population. Each solution in the population is called a chromosome, which represents a point in the search space. The genetic search progress is obtained by two essential genetic operations: exploitation (or intensification) and the exploration (or diversification). Generally, the crossover operator exploits a better solution while the mutation operator explores a wider search space.

The chromosomes evolve through successive iterations, called generations. During each generation, the chromosomes are evaluated by using some measures of fitness. The fitter the chromosomes, the higher the probabilities of being selected to perform a genetic operation (either crossover or mutation). In the crossover phase, the GA attempts to exchange portions of two parents (two chromosomes in the population) to generate an offspring. The crossover operation speeds up the process to reach better solutions. In the mutation phase, the mutation operation maintains the diversity in the population to avoid being trapped in local optima. A new generation is formed by selecting some of the parents and offspring according to their fitness values, and by rejecting others so to keep the population size constant. After the predetermined number of generations is performed, the algorithm converges to the best chromosome, which hopefully represents the optimal solution or may be a sub-optimal solution to the problem. 
As mentioned before, a GA has a great level of flexibility. It can be hybridized with other heuristics in order to improve the solution further. However, it was found that none of the researchers have studied the PAP problem using a hybrid GA (HGA). In this paper, a HGA is proposed to solve the PAP problem. The flowchart of the HGA for the PAP problem is shown in Fig. 1. The procedure of the HGA for the PAP problem is listed as follows:

Step 1: Set the GA parameters, including the population size (psize), the number of iterations (itno), the crossover rate ( $c r)$, and the mutation rate $(\mathrm{mr})$.

Step 2: $\quad$ Generate psize initial chromosomes with two-link encoding to be discussed in Section 3.1. For each chromosome, the first link is generated by the nearest neighbor heuristic $(\mathrm{NNH})$ as shown in Section 3.2.1 while the second link is generated randomly.

Step 3: The 2-opt local search heuristic to be presented in Section 3.2.2 is performed on the second link for each initial chromosome.

Step 4: The iterated swap procedure (ISP) addressed in Section 3.2.3 is performed on the first link for each initial chromosome.

Step 5: $\quad$ Evaluate the fitness value $\operatorname{eval}\left(X_{h}\right)$ as illustrated in Section 3.3 for all chromosomes in the population.

Step 6: Follow the selection procedure in Section 3.4 to select chromosomes to perform the modified order crossover operation in Section 3.5.1.

Step 7: Follow the selection procedure to select chromosomes to perform the heuristic mutation operation in Section 3.5.2.

Step 8: Follow the selection procedure to select chromosomes to perform the inversion operation in Section 3.5.3.

Step 9: The 2-opt local search heuristic is performed on the second link for each offspring generated in Steps 6, 7, 8. 
Step 10: The ISP is performed on the first link for each offspring generated in Steps 6, 7, 8.

Step 11: Compare all offspring with the chromosomes in the population by the fitness values eval $\left(X_{h}\right)$. Retain the best psize chromosomes in the population.

Step 12: Determine the best chromosome at each iteration. Repeat Step 6 to Step 12 until itno iterations are performed.

After the parameters have been set up (Step 1), the HGA generates an initial population in which the first links in the chromosomes, representing the sequence of the component placements, are generated from the $\mathrm{NNH}$ while the second links, representing the feeder arrangement, are generated randomly (Step 2). During this initialization step, each chromosome is improved as follows: the 2-opt local search heuristic is applied to the second link (Step 3) while the ISP, which is a new improving heuristic, is performed on the first link (Step 4). Each chromosome is then measured by an evaluation function (Step 5). The roulette wheel selection operation is performed to select some chromosomes for the genetic operations. These operations consist of one crossover and two mutations (Steps 6, 7, and 8). After an offspring is produced, the second links are improved by the 2-opt local search heuristic (Step 9) while the first link is improved by the ISP (Step 10). The fitness of the offspring will be measured and may become a member of the population if it possesses a relatively good quality (Step 11). These steps form an iteration, and then the roulette wheel selection is performed again to start the next iteration. The HGA will not stop unless the predetermined number of iterations is conducted (Step 12).

\subsection{Encoding}

A chromosome is illustrated in Fig. 2 with the two-link representation, in which the number inside the bracket in link 1 represents the component type. The path representation is used to encode the solution to the PAP problem. The idea of the path representation is that the 
components are listed in the order in which they are placed. For example, suppose there are 5 components numbered as $1,2,3,4$, and 5 . If the sequence of placements starts with component

3 , then component 1 , component 4 , component 5 , and finally component 2 , its sequence can be

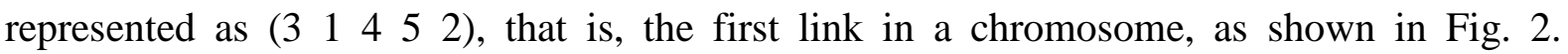
Furthermore, if the five components are from three component types, then component 3 of type 2 is placed first, whereas the component 2 of type 2 is placed last. On the other hand, component type 2 is located at feeder 1 . Therefore, the placement head has to visit feeder 1 , picks up a component of type 2, then places it on the board for component 3 .

As a consequence, the sequence of component placements, $c$, will be (3 145 2), while the sequence of the feeders to be visited by the placement head, $f$, will be (1 2223 1). The entire assembly sequence of the placement head, illustrated in Fig. 3, will be: starting point $\rightarrow f_{1} \rightarrow c_{3}$ $\rightarrow f_{2} \rightarrow c_{1} \rightarrow f_{2} \rightarrow c_{4} \rightarrow f_{3} \rightarrow c_{5} \rightarrow f_{1} \rightarrow c_{2} \rightarrow$ starting point.

\subsection{Improving heuristics}

Although the PAP problem is simple to describe verbally, it is very difficult to solve optimally when the number of components or the problem size is large. The improving heuristics are therefore helpful to tackle with this problem. In the HGA, three types of heuristics are adopted to improve the solution, including the nearest neighbor heuristic, the 2-opt local search heuristic, and the iterated swap procedure.

\subsubsection{The nearest neighbor heuristic}

The Nearest Neighbor Heuristic (NNH) is used to generate an initial solution for the first link only, which is the sequence of the component placements. The principle of the NNH is to start with the first component randomly, then to select the next component as close as possible to the previous one from those unselected components to form the placement sequence until all 
components are selected.

\subsubsection{The 2-opt local search heuristic}

Comparing with the total number of components in a PCB, the number of different component types is much fewer. Moreover, the number of feeders in a PAP machine is very limited. Therefore, it is desirable to perform the 2-opt local search heuristic for the second link only, that is, the feeder arrangement. The principle of this heuristic is very straightforward. For one parent, all possible 2 swaps are examined to generate offspring and the best offspring will replace the parent if the offspring has a shorter traveling distance than the parent.

\subsubsection{The iterated swap procedure}

The computational effort will be high if the 2-opt local search is performed on the first link, which is the sequence of component placements, because the number of components is quite large, normally several hundreds. As a consequence, a 'fast' improving heuristic is developed, which is called the iterated swap procedure (ISP). The ISP, illustrated in Fig. 4, is performed for the first link of each initial solution generated by the $\mathrm{NNH}$ as well as each offspring generated by the three genetic operators. The procedure of the ISP is as follows:

Step 1: $\quad$ Select two genes randomly from the first link of a parent.

Step 2: $\quad$ Exchange the positions of the two genes to form an offspring.

Step 3: $\quad$ Swap the neighbors of the two genes to form four more offspring.

Step 4: $\quad$ Evaluate all offspring and find the best one.

Step 5: If the best offspring is better than the parent, replace the parent with the best offspring and go back to Step 1; Otherwise, Stop.

\subsection{Evaluation}

For the PAP problem, the fitness function used is the total traveling distance of the placement 
head. This function calculates the total distance the head traveled. It includes the distance from the original location (starting point) to a feeder at the beginning, the distances from a feeder to a component, the distances from a component to a feeder, and the distance from the last component to the original location (starting point). Let eval( $\left.X_{h}\right)$ be the fitness function for chromosome $X_{h}$ in the PAP problem, and $D(a, b)$ represent the distance from point $a$ to point $b$, then

$$
\operatorname{eval}\left(X_{h}\right)=D(o, f(1))+\sum_{i=1}^{n} D\left(f(i), c_{i}\right)+\sum_{i=1}^{n-1} D\left(c_{i}, f(i+1)\right)+D\left(c_{n}, o\right)
$$

where

$n$ is the number of components, and

$f(i)$ is the feeder location for the $i$ th component, and

$c_{i}$ is the location in the board for the ith component, and

$o$ is the original location or starting point.

Here, $f(i)$ represents the feeder location for the ith component. For example, for component 3 , it is stored in Feeder 1 , so $f(3)=1$, that is, $f_{1}$ in the previous discussion.

\subsection{Selection}

The roulette wheel approach is adopted in order to choose some chromosomes to undergo genetic operations. The approach is based on an observation that a roulette wheel has a section allocated for each chromosome in the population, and the size of each section is proportional to the chromosome's fitness. The fitter the chromosome, the higher the probability of being selected. It is true that the roulette wheel selection mechanism chooses chromosomes probabilistically, instead of deterministically. For example, although one chromosome has the highest fitness, there is no guarantee it will be selected. The only thing that is certain is that on the average a chromosome will be chosen with the probability proportional to its fitness. Suppose the population size is psize, then the selection procedure is as follows: 
Step 1: Calculate the total fitness of the population:

$$
F=\sum_{h=1}^{\text {psize }} \operatorname{eval}\left(X_{h}\right)
$$

Step 2: $\quad$ Calculate the selection probability $p_{h}$ for each chromosome $X_{h}$ :

$$
p_{h}=\frac{F-\operatorname{eval}\left(X_{h}\right)}{F \times(p s i z e-1)}, \quad h=1,2, \ldots, p \text { size }
$$

Step 3: $\quad$ Calculate the cumulative probability $q_{h}$ for each chromosome $X_{h}$ :

$$
q_{h}=\sum_{j=1}^{h} p_{j}, \quad h=1,2, \ldots, \text { psize }
$$

Step 4: $\quad$ Generate a random number $r$ in the range $(0,1]$.

Step 5: $\quad$ If $q_{h-1}<r \leq q_{h}$, then chromosome $X_{h}$ is selected.

\subsection{Genetic operations}

The genetic operators used in the algorithm for the PAP problem are one crossover and two mutations, which are called the heuristic mutation and the inversion mutation, respectively. The two links in a chromosome are required to perform these genetic operations. The number of chromosomes selected to perform the crossover and mutation operations depends on the crossover rate and the mutation rate, respectively, which are preset by the GA user. Let crossno and mut denote the number of chromosomes selected to undergo the crossover and the mutation, respectively, then $c r o s s n o=\operatorname{round}(c r \times p s i z e)$ and $m u t=\operatorname{round}(m r \times p s i z e)$, where $c r$ is the crossover rate, and $m r$ is the mutation rate. Since a pair of chromosomes is required to undergo the crossover operation, the number of pairs of chromosomes, denoted as cross, is an integer, so 


$$
\text { cross }= \begin{cases}\frac{\text { crossno }}{2} & \text { if crossno is even } \\ \frac{\text { crossno }-1}{2} & \text { otherwise }\end{cases}
$$

\subsubsection{The modified order crossover operation}

As shown in Fig. 5, the crossover operator adopted in the HGA is a modified version of the classical order crossover operator, and two offspring will be generated at each time.

The procedure of the modified order crossover operation is listed as follows:

Step 1: $\quad$ Select a substring from the first parent randomly.

Step 2: $\quad$ Produce a proto-child by copying the substring into the corresponding positions in the proto-child.

Step 3: Find the gene right prior to the first gene of the substring from the second parent. If the gene is one of the genes in the substring, go to Step 4. Otherwise, place it in front of the substring in the proto-child.

Step 4: $\quad$ Find the gene right behind the last gene of the substring from the second parent. If the gene is one of the genes in the substring, go to Step 5. Otherwise, place it just after the substring in the proto-child.

Step 5: Delete those genes that are already in the proto-child from the second parent. The resulted genes, that is, the genes not in the proto-child yet, form a sequence.

Step 6: Place the genes into the unfilled positions of the proto-child from the left to the right according to the resulted sequence of genes in Step 5 to produce an offspring.

Step 7: $\quad$ Repeat Step1 to Step 6 to produce the second offspring by exchanging the two parents.

\subsubsection{The heuristic mutation operation}

A heuristic mutation is designed with the neighborhood technique in order to produce a better offspring. A set of chromosomes transformed from a parent by exchanging some genes is 
regarded as the neighborhood. Only the best one among the neighborhood is used as the offspring produced by the mutation. However, the purpose of the mutation operation is to promote diversity of the population. Therefore, it is necessary to make change to the original heuristic mutation for the PAP problem. The modification, illustrated in Fig. 6, is that all neighbors generated are used as the offspring. The procedure of the heuristic mutation operation is listed as follows:

Step 1: $\quad$ Pick up 3 genes in a parent at random.

Step 2: Generate neighbors for all possible permutations of the selected genes, and all neighbors generated are regarded as the offspring.

Here, in Step 1, only 3 genes are selected since 2 genes has only one variation (one offspring) while more than 3 genes will generate too many offspring and it will take a very long time for computation.

\subsubsection{The inversion operation}

It can be noticed the genetic algorithm developed especially for the PCB assembly problems is not limited to using one crossover and one mutation. Due to the complexity of the problems, several genetic operations will be adopted (Leu et al., 1993; Ong and Khoo, 1999). In this paper, two mutation operations are adopted. Apart from the heuristic mutation, the inversion is the second mutation to be adopted. The inversion operator, as shown in Fig. 7, selects a substring from a parent and flips it to form an offspring. Since the inversion operator operates with one chromosome very like the heuristic mutation and thus lacks interchange of the characteristics between chromosomes. So, the inversion operator is a mutation operation, which is used to increase the diversity of the population rather than to enhance the quality of the population. 


\section{Performance analysis}

The performance of the HGA was evaluated by use of the PCB example in (Leu et al., 1993). The example has 200 components with 10 different component types. The parameters of the HGA for the problem are preset as: population size psize $=25$, iteration number itno $=3000$, crossover rate $c r=0.4$, and mutation rate $m r=0.2$. Therefore, 5 pairs of chromosome are selected to perform the modified order crossover operation, whereas 5 chromosomes perform the heuristic mutation and the inversion operation. The total number of offspring produced per iteration will be 40, 10 from the modified order crossover operation, 25 from the heuristic mutation, and 5 from the inversion operation. The performance of the HGA is shown in Fig. 8, whereas the comparison between the results obtained from the HGA and those obtained from other researchers is shown in Table 1.

According to Fig. 8 and Table 1, it is found that the performance of the HGA is superior to that of the simple GAs used by Leu et al. (1993) as well as Ong and Khoo (1999) in four aspects. Firstly, the best chromosome $(6,275.4 \mathrm{~cm})$ in the initial population obtained by the HGA is better than that of the simple GAs (both larger than 6,900 cm). Secondly, the HGA can obtain a better solution with a smaller population size, 25 only, while the other two used 100 . Thirdly, the HGA can obtain a better solution not only with a smaller population size, but also with a fewer iterations, 3000 vs. 6150 . Finally and the most importantly, the HGA obtained a better solution than any previous methods, $5660.5 \mathrm{~cm}$ vs. $5673.7 \mathrm{~cm}$ or $6129 \mathrm{~cm}$.

\section{Conclusions}

A hybrid genetic algorithm incorporated with three different heuristics was applied successfully to the PAP problem, which is the combined problem of component sequencing 
and feeder arrangement in PCB assembly with the objective of minimizing the total traveling distance of the placement head for a pick and placement machine. The three heuristics are the nearest neighbor heuristic, the 2-opt local search heuristic, and a newly developed improving heuristic called the iterated swap procedure. Furthermore, a two-link representation together with the modified order crossover, the heuristic mutation, and the inversion mutation was used to solve the problem. Finally, it was shown that the performance of the HGA is superior to that of the simple GAs in Leu et al. (1993) and Ong and Khoo (1999) in terms of the total distance traveled by the placement head.

\section{Acknowledgements}

The authors wish to acknowledge The Hong Kong Polytechnic University for the financial support of the project (No. G-W031). 


\section{References}

Altinkemer, K., Kazaz, B., Köksalan, M. and Moskowitz, H. (2000) Optimization of printed circuit board manufacturing: Integrated modeling and algorithms. European Journal of Operational Research, 124, 409-421.

Ball, M. O. and Magazine, M. J. (1988) Sequencing of insertions in printed circuit board assembly. Operations Research, 36(2), 192-201.

Broad, K., Mason, A., Rönnqvist, M. and Frater, M. (1996) Optimal robotic component placement. Journal of Operational Research Society, 47, 1343-1354.

Chambers, L. (1995) Practical Handbook of Genetic Algorithms, Volume II, CRC press, Boca Raton, Florida.

Chan, D. and Mercier, D. (1989) IC insertion: an application of the travelling salesman problem. International Journal of Production Research, 27(10), 1837-1841.

Davis, L. (1991) Handbook of Genetic Algorithms, Van Nostrand Reinhold, New York.

Foulds, L. R. and Hamacher, H. W. (1993) Optimal bin location and sequencing in printed circuit board assembly. European Journal of Operational Research, 66, 279-290.

Francis, R. L., Hamacher, H. W., Lee, C. Y. and Yeralan, S. (1994) Finding placement sequences and bin locations for cartesian robots. IIE Transactions, 26(1), 47-59.

Gen, M. and Cheng, R. (1997) Genetic Algorithms and Engineering Design, John Wiley \& Sons, New York.

Ji, Z., Leu, M. C. and Wong, H. (1992) Application of linear assignment model for planning of robotic printed circuit board assembly. Journal of Electronic Packaging, 114, 455-460.

Kumar, R. and Li, H. (1995) Integer programming approach to printed circuit board assembly time optimization. IEEE Transactions on Components, Packaging, and Manufacturing Technology - Part B, 18(4), 720-727.

Leu, M. C., Wong, H. and Ji, Z. (1993) Planning of component placement/insertion sequence 
and feeder setup in PCB assembly using genetic algorithm. Journal of Electronic Packaging, 115, 424-432.

Magyar, G., Johnsson, M. and Nevalainen, O. (1999) On solving single machine optimization problems in electronics assembly. Journal of Electronics Manufacturing, 9(4), 249-267.

Mitchell, M. (1996) Introduction to Genetic Algorithms, The MIT Press, Cambridge, Massachusetts.

Ong, N. S. and Khoo, L. P. (1999) Genetic algorithm approach in PCB assembly. Integrated Manufacturing Systems, 10, 256-265.

Reinelt, G. (1994) The Traveling Salesman: Computational Solutions for TSP Applications, Springer-Verlag, Berlin, New York. 
Table 1. A comparison of the experimental results

\begin{tabular}{|lccc|}
\hline & Leu et al. (1993) & Ong and Khoo (1999) & HGA \\
Population size & 100 & 100 & 25 \\
Iteration number & 6,150 & 6,150 & 3,000 \\
Total distance $(\mathrm{cm})$ & about 6,129 & $5,673.7$ & $5,660.5$ \\
\hline \hline
\end{tabular}




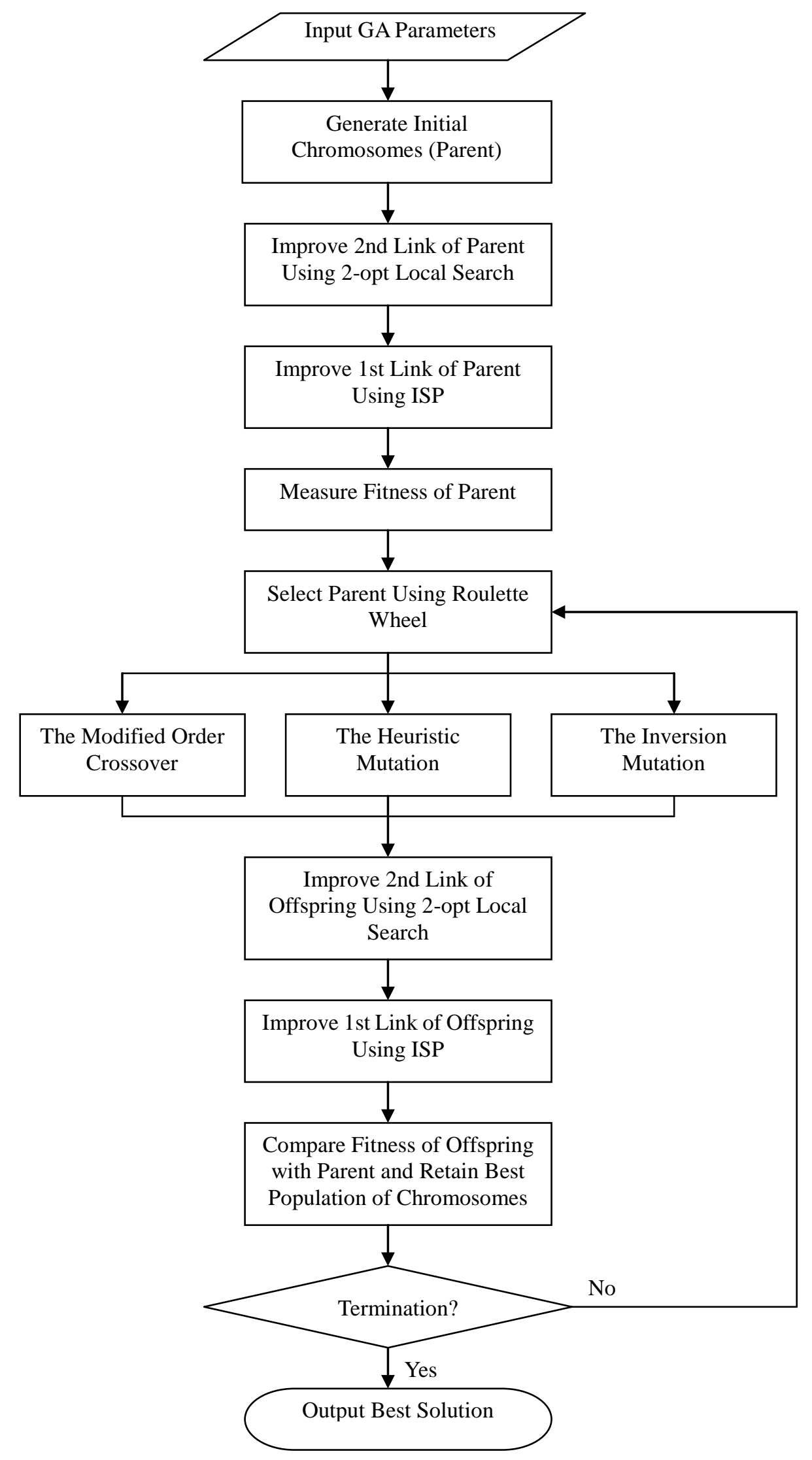

Fig. 1. The flowchart of the HGA. 


\begin{tabular}{|c|c|c|c|c|c|}
\hline Assembly Sequence & 1 & 2 & 3 & 4 & 5 \\
\hline Component Number & $3(2)$ & $1(3)$ & $4(3)$ & $5(1)$ & $2(2)$ \\
\hline
\end{tabular}

Component Type

Feeder

\begin{tabular}{|l|l|l|}
\hline 2 & 3 & 1 \\
\hline 1 & 2 & 3
\end{tabular}

Link 2

Fig. 2. The two-link representation for a chromosome. 


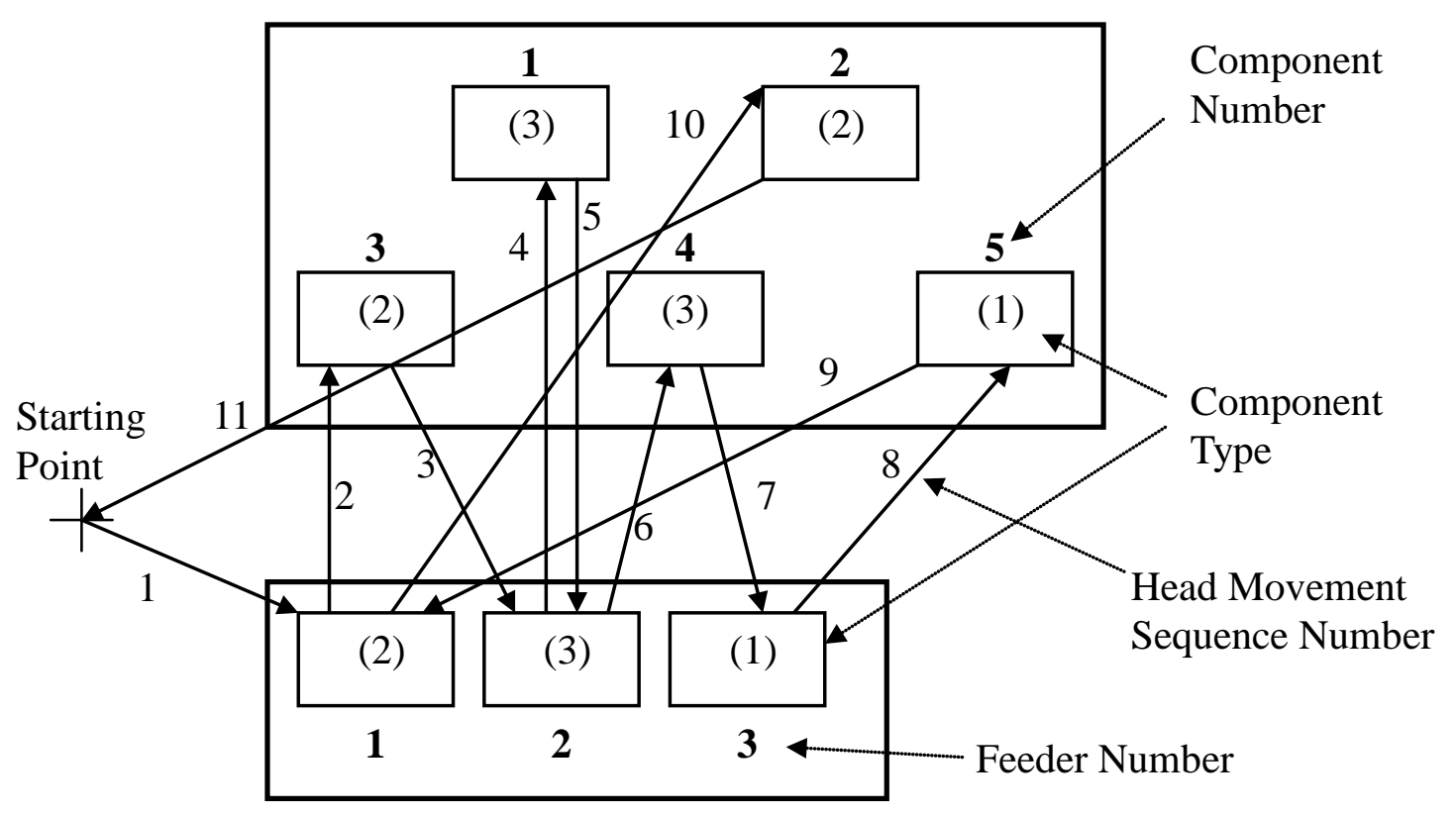

Fig. 3. The assembly sequence of the placement head. 
Select 2 genes randomly

Parent:

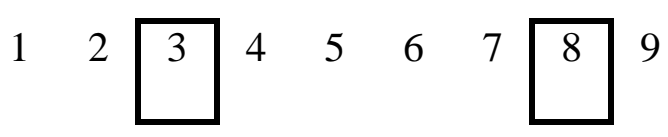

Offspring 1:

$\begin{array}{lllllllll}1 & 2 & 8 & 4 & 5 & 6 & 7 & 3 & 9\end{array}$

Offspring 2:

$\begin{array}{lllllllll}1 & 8 & 2 & 4 & 5 & 6 & 7 & 3 & 9\end{array}$

Offspring 3:

$\begin{array}{lllllllll}1 & 2 & 4 & 8 & 5 & 6 & 7 & 3 & 9\end{array}$

Offspring 4:

$\begin{array}{lllllllll}1 & 2 & 8 & 4 & 5 & 6 & 3 & 7 & 9\end{array}$

Offspring 5:

$\begin{array}{lllllllll}1 & 2 & 8 & 4 & 5 & 6 & 7 & 9 & 3\end{array}$

Fig. 4. The iterated swap procedure. 
Selected substring

Parent 1:

Parent 2:

$\begin{array}{lllllllll}1 & 2 & 3 & 4 & 5 & 6 & 7 & 8 & 9 \\ 5 & 7 & 4 & 9 & 1 & 3 & 6 & 2 & 8\end{array}$

Offspring 1:

$\begin{array}{lllllllll}7 & 1 & 3 & 4 & 5 & 6 & 2 & 9 & 8\end{array}$

Parent 2:

Parent 1:

\begin{tabular}{llllll|llll}
5 & 7 & 4 & 9 & 1 & 3 & 6 & 2 & 8 \\
1 & 2 & 3 & 4 & 5 & 6 & 7 & 8 & 9
\end{tabular}

Offspring 2:

$\begin{array}{lllllllll}2 & 5 & 4 & 9 & 1 & 3 & 6 & 7 & 8\end{array}$

Fig. 5. The modified order crossover operator. 
Select 3 genes at random

\begin{tabular}{|c|c|c|c|c|c|c|c|c|}
\hline Parent: & 1 & 2 & 3 & 4 & 5 & 6 & 7 & 8 \\
\hline Offspring 1: & 1 & 2 & 3 & 4 & 5 & 8 & 7 & 6 \\
\hline Offspring 2: & 1 & 2 & 6 & 4 & 5 & 3 & 7 & 8 \\
\hline Offspring 3: & 1 & 2 & 6 & 4 & 5 & 8 & 7 & 3 \\
\hline Offspring 4: & 1 & 2 & 8 & 4 & 5 & 3 & 7 & 6 \\
\hline Offspring 5: & 1 & 2 & 8 & 4 & 5 & 6 & 7 & 3 \\
\hline
\end{tabular}

Fig. 6. The heuristic mutation operator. 
Selected substring

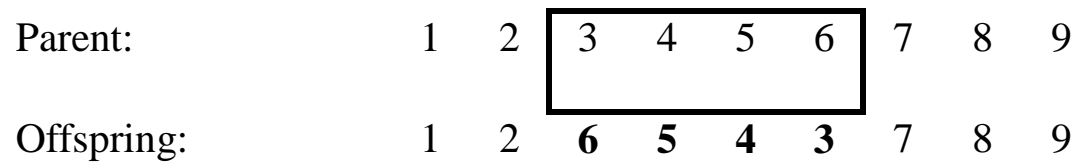

Fig. 7. The inversion operator. 
Total traveling distance $(\mathrm{cm})$

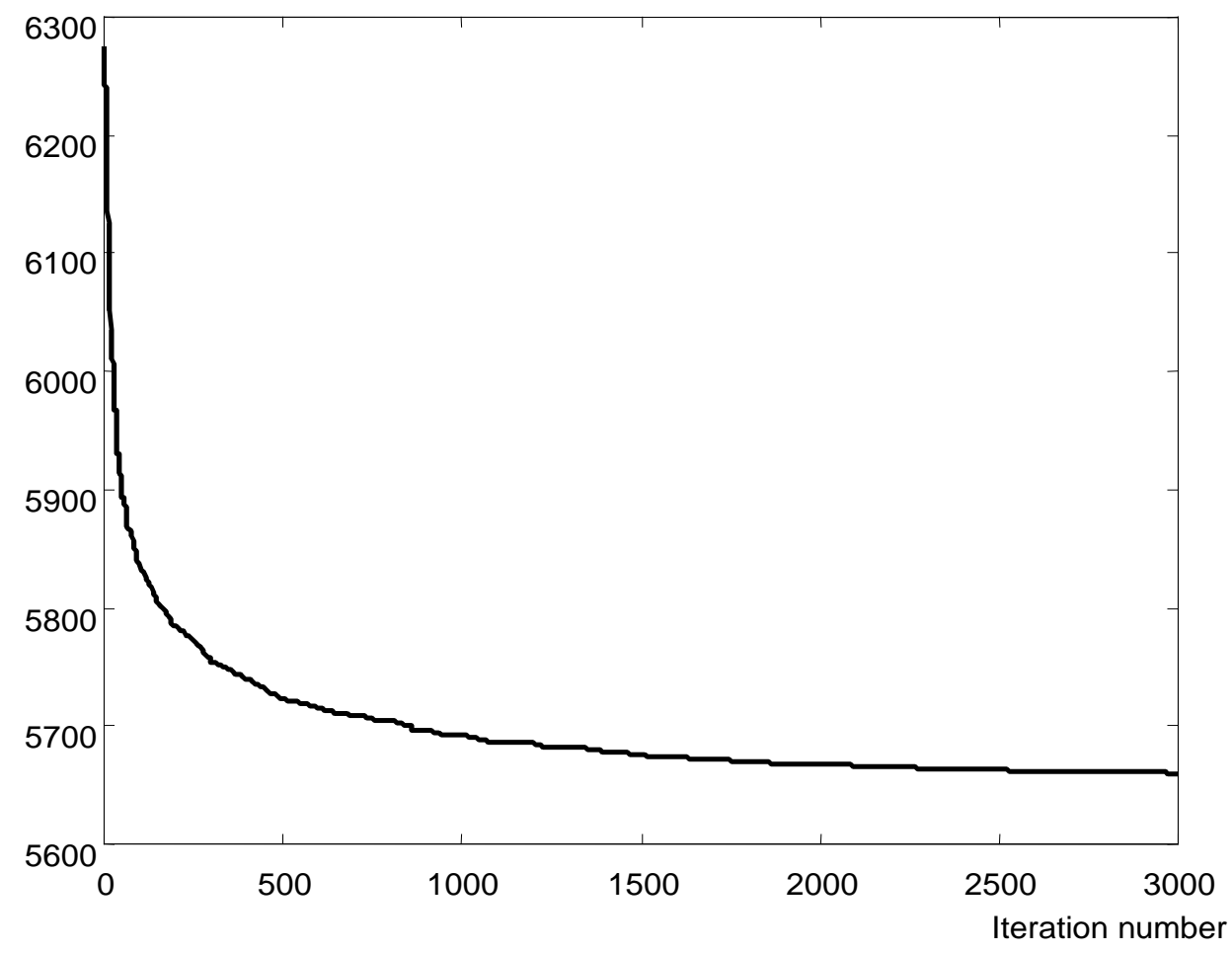

Fig. 8. The minimum traveling distance at each iteration. 
Fig. 1. The flowchart of the HGA.

Fig. 2. The two-link representation for a chromosome.

Fig. 3. The assembly sequence of the placement head.

Fig. 4. The iterated swap procedure.

Fig. 5. The modified order crossover operator.

Fig. 6. The heuristic mutation operator.

Fig. 7. The inversion operator.

Fig. 8. The minimum traveling distance at each iteration. 


\section{University Library}

\section{- M M I N E R VA A gateway to Melbourne's research publications}

Minerva Access is the Institutional Repository of The University of Melbourne

Author/s:

Ho, W;Ji, P

Title:

A hybrid genetic algorithm for component sequencing and feeder arrangement

Date:

2004-06-01

Citation:

Ho, W. \& Ji, P. (2004). A hybrid genetic algorithm for component sequencing and feeder arrangement. JOURNAL OF INTELLIGENT MANUFACTURING, 15 (3), pp.307-315. https:// doi.org/10.1023/B:JIMS.0000026569.88191.46.

Persistent Link:

http://hdl.handle.net/11343/118697 